\section{DIFFERENCES IN IMMUNE-RELATED ADVERSE EVENTS BETWEEN VULVOVAGINAL VS. CUTANEOUS MELANOMA: A RETROSPECTIVE COHORT STUDY}

Sheida Naderi-Azad. University of Toronto Faculty of Medicine

\subsection{6/ijgc-2020-ESG0.180}

Introduction/Background Few studies to date have comprehensively examined all immune-related adverse events (irAEs) in vulvovaginal and cutaneous melanoma patients on immune checkpoint inhibitors (ICIs).

Methodology We retrospectively analyzed 169 patients with advanced-stage vulvovaginal or cutaneous melanoma who received at least one dose of ICI between June 2012 to December 2018. Descriptive statistics were used to summarize the baseline characteristics, disease outcomes, and toxicity profiles. Chi-square statistical analysis was used to examine associations between irAEs and pre-existing conditions, as well as irAEs and treatment response. P-values $<0.05$ were considered statistically significant.

Results Overall, 53.8\% of patients with vulvovaginal melanoma developed irAEs, compared to a similar percentage of $51.9 \%$ for patients with cutaneous melanoma. Yet the most common types of irAEs differed between patients. The most common irAEs for patients with vulvovaginal melanoma were gastrointestinal disorders (44.4\%), hypothyroidism (22.2\%), and renal and urinary disorders (22.2\%). On the other hand, the most common irAEs for patients with cutaneous melanoma on ICIs were gastrointestinal disorders (21.7\%), cutaneous adverse events (17.9\%) and pneumonitis (18.75\%). Cutaneous adverse events were overall the most common irAEs, and were significantly associated with patient response to ICIs $(\mathrm{p}=0.01)$.

Conclusion Nuanced differences in the clinical presentation of irAEs in patients with vulvovaginal vs. cutaneous melanoma are important considerations for initiating ICIs in accordance with melanoma type. Furthermore, cutaneous adverse events were the most common irAEs overall, and were significantly associated with response to ICIs in patients with metastatic melanoma.

Disclosures I have no conflicts of interest.

\section{THE EFFICACY OF FIBRIN SEALANTS IN REDUCING THE POST-OPERATIVE MORBIDITY AFTER INGUINO-FEMORAL LYMPHADENECTOMY IN MELANOMA AND VULVAL CANCER: BACK TO THE DRAWING BOARD?}

${ }^{1}$ Anastasios Tranoulis, ${ }^{2}$ Dimitra Georgiou, ${ }^{3}$ Bindiya Gupta, ${ }^{4}$ Omer Devaja, ${ }^{4}$ Stephen AttardMontalto. 'The Pan-Birmingham Gynaecological Oncology Centre, Sandwell and West Birmingham NHS Trust, Birmingham; ${ }^{2}$ Chelsea and Westminster NHS Foundation Trust, Imperial College; ${ }^{3}$ The Pan-Birmingham Gynaecological Oncology Centre, City Hospital; Panbirmingham Gynaecological Cancer Centre, Birmingham City Hospital; Gynaecological Oncology: ${ }^{4}$ Maidstone and Tunbridge Wells NHS Trust, Maidstone, UK

\subsection{6/ijgc-2020-ESGO.181}

Introduction/Background The inguino-femoral lymphadenectomy (IFL) and its associated morbidity remains to date a field of contention. Several intra-operative techniques have been advocated to intimidate the IFL-related complications. Despite the improvements in the IFL technique, apprehension towards operative complications still exists. The intra-operative application of collagen-fibrin sealants (CFS) has emerged as a promising intervention to reduce the IFL-related lymphorrhoea. To ascertain the clinical utility of CFS during IFL, we performed a meta-analysis to draw conclusions about their efficacy with the primary objective of reducing the volume and the duration of lymphatic drainage. A secondary objective was to elucidate its effectiveness in reducing other wound complications.

Methodology MEDLINE, Scopus and Cochrane Database were searched for relevant references from inception until August 2020 in line with PRISMA guidelines. Randomized controlled studies (RCTs) and observational studies (OSs) comparing the post-operative morbidity after IFL with or without the use of CFS were included. The modified Jadad score and the methodologic index for non-randomized studies were used to evaluate the quality of the included studies. Dichotomous variables were assessed using odds ratio (OR), whilst continuous variables were assessed using the standartised mean difference (SMD). Confidence intervals were set at 95\%. The DerSimonian-Laird random-effects model was used due to the expected inter-study heterogeneity. Statistical analysis was performed using the RevMan software version 5.3. The level of statistical significance was set at p-value $<0.05$.

Results Six RCTs and four OSs encompassing 305 and 221 patients respectively were included. The studies were of moderate quality and characterised by significant clinical heterogeneity. The meta-analysis of RCTs demonstrated that the application of CFS did neither decrease the length of drainage [SDM -0.55 (95\% CI -1.34 to 0.23$), \mathrm{p}=0.17]$ nor the amount of drained output [SMD $0.46 \quad(95 \%$ CI -0.29 to 1.20$)$, $\mathrm{p}=0.23]$. No significant difference was found regarding the incidence of lymphocele(s) formation [OR 0.96 (95\% CI 0.56 - 1.65), $\mathrm{p}=0.88$ ] or other wound complications. The safety profile of CFS was favourable. No severe adverse sequelae were reported.

Conclusion Our findings suggest that the use of CFS was not associated with difference in the incidence of IFL-related lymphorrhoea. Their safety profile was favourable. This evidence is constrained by the data available with an inevitable emphasis on short-term outcomes. In view of the lack of clinical equipoise, more high quality RCTs are warranted to draw firmer conclusions. An attempt should be made at standardising outcome measures, which will improve comparability between studies.

Disclosures None.

\section{ROBOTIC INGUINOFEMORAL GROIN NODE DISSECTION - MODIFICATIONS TO EXISTING ROBOTIC TECHNIQUE}

${ }^{1}$ Ts Shylasree, ${ }^{2}$ Pooja Singh. 'Tata Memorial Hospital; Department of Gynecologic Oncology and Mdt; ${ }^{2}$ Tata Memorial Hospital; Gynecological Oncology

\subsection{6/ijgc-2020-ESG0.182}

Introduction/Background Techniques to improve problems encountered during robotic Inguino-femoral node dissection and prevent muscle miss during surgery

Methodology Identification of muscles in the anterior upper half of the thigh is of paramount importance for approaching femoral triangle and femoral blood vessels. Sartorius and Vastus medialis are almost in the same spot in the anterior middle third of the thigh during dissection. Problems encountered in the initial five robotic groin node dissections with regards to anatomical muscle miss were addressed by 
rearranging the position of the ports and bringing them closer to the tip of the femoral triangle. A 30 degree telescope helped in visualising the tip of the triangle better to remove the nodal tissue enbloc at completion of surgery.

Results Improved techniques led to easy identification of sartorius and standardization of the procedure.

Conclusion Issues and tips for improvement in surgical techniques especially in novel areas like robotic Inguino-femoral node dissection surgery are addressed.

Disclosures This surgical video was presented at IGCS Conference 2019.

\section{INTRA-TUMOURAL ELECTRO-CHEMOTHERAPY (ECT) WITH BLEOMYCIN FOR PALLIATION OF CUTANEOUS RECURRENCE IN GYNAECOLOGICAL MALIGNANCY}

${ }^{1}$ Stuart Rundle, ${ }^{1}$ Christine Ang, ${ }^{2}$ Tani Fasih, ${ }^{1}$ Porfyrios Korompelis, ${ }^{1}$ Nithya Ratnavelu. ${ }^{1}$ Northern Gynaecological Oncology Centre; Queen Elizabeth Hospital; ${ }^{2}$ Queen Elizabeth Hospital; Breast and General Surgery

\subsection{6/ijgc-2020-ESGO.183}

Introduction/Background ECT utilises pulsed electrical current to transiently increase cell membrane permeability to the cytotoxic agent, bleomycin. We present the use of ECT in patients with recurrent gynaecological malignancy previously treated with a combination of surgery or radiotherapy, experiencing symptoms from cutaneous lesions. This report presents evidence of the role for ECT in second and third line treatment. Methodology Between July 2017 and August 2019, 6 patients with cutaneous recurrence of gynaecological malignancy (5 vulvar SCC and 1 high grade serous ovarian cancer) were treated with intra-tumoural bleomycin (9000 iu or $15000 \mathrm{iu}$, dependent on tumour volume) and pulsed-probe electroporation. Response was assessed clinically in routine follow up or following self-referral with return of symptoms. Post procedure pain scores were collated as part the quality of life evaluation.

Results Median treated tumour diameter was $6 \mathrm{~cm}$ (range 2 $12 \mathrm{~cm}$ ). Pain scores peaked between day 2 and day 7 postprocedure. The median progression free interval was 3.6 months (range $0.8-6.7$ months).

Following ECT treatment 2 patients continued to receive supportive care. Two patients underwent repeat treatment with ECT and reported symptom improvement with each treatment. Due to further progression two patients underwent radical surgery and one patient received palliative chemotherapy.

Conclusion ECT should be considered for patients with symptomatic cutaneous recurrence of gynaecological malignancy who have previously had multi-modal treatment. It can achieve symptom control and reduce the need for radical surgery in this palliative setting.

Disclosures The Authors have no conflicts of interest to disclose.

\section{NEW VARIANT OF RECONSTRUCTIVE SURGERY FOR ADVANCED VULVAR CANCER TREATMENT}

${ }^{1}$ Olha Bubliieva, ${ }^{2}$ Yevgeniy Kostiuchenko, ${ }^{1}$ Valentyn Svintsitskiy, ${ }^{3}$ Igor Motuziuk, ${ }^{1}$ Nataly Tsip, ${ }^{1}$ Sergiy Nespryadko, ${ }^{1}$ Alena Samokhvalova, ${ }^{3}$ Oleg Sydorchuk. ${ }^{1}$ National Cancer Institute; ${ }^{2}$ National Cancer Institute; 0.0. Bogomolets National Medical University; ${ }^{3} 0.0$. Bogomolets National Medical University

10.1136/ijgc-2020-ESGO.184
Introduction/Background Surgical treatment of advanced vulvar malignant tumors usually requires immediate reconstruction. Large defects after pelvis, vagina, vulva, groin and perineum wide excision require closure with the usage of difficult reconstructive techniques. In this case the most suitable myocutaneous flap for reconstruction is rectus abdominis muscle flap, which provides the biggest volume of tissues to cover those large defects.

Methodology Woman 67 y.o. initially presented with the combined treatment of cervical cancer stage IIB. Within 5 month was diagnosed the lymphedema in the left lower extremity. Approximately 2 years she presented the vulvar tumor measured $15 \times 10 \mathrm{~cm}$. A biopsy was performed the lymphangiosarcoma.

Results In the National Cancer Institute of Ukraine we investigated a new variation of large defects reconstruction using rectus abdominis muscle flap. To collect a donor flap, we perform 3 arcuate incisions: one vertical by the medial line around the umbilicus, and two oblique incisions towards upper anterior iliac spine of one of the sides. In this way we use only one half of the abdomen, and in case of any complications with the flap or relapse of the disease we will have the second donor site for possible future reoperation. There is a narrow 'bridge' of tissues we leave between the excised donor and recipient sites. It is extremely important to preserve blood supply not only to the flap, but also to the 'bridge' to avoid complications. We perform a tunnel under the 'bridge' as small as possible to preserve all inferior epigastric vessels, but enough to transfer the flap and not to squeeze the pedicle. After the surgery we have a half-inverted Y-shaped scar on of the sides of the abdomen, an inverted triangular scar at the pelvis area, circumumbilical scar and a short vertical scar on the flap to imitate pudendal cleft with central structures of vulva (urethra and vaginal tube).

Conclusion We consider our variation of this type of surgery the most safe and efficient, with the opportunity of re-operation if needed. Advanced vulvar malignancies are quite rare, so we will keep working on development and enhancement of the technique to help these patients.

Disclosures Authors declare no disclosures.

\section{VULVAR RECONSTRUCTION IN PATIENTS WITH ADVANCED MALIGNANCIES}

${ }^{1}$ Olha Bubliieva, ${ }^{2}$ Yevgeniy Kostiuchenko, ${ }^{3}$ Igor Motuziuk, ${ }^{1}$ Valentyn Svintsitskiy, ${ }^{1}$ Sergiy Nespryadko, ${ }^{1}$ Nataly Tsip, ${ }^{3}$ Oleg Sydorchuk, ${ }^{1}$ Alena Samokhvalova. ${ }^{1}$ National Cancer Institute; ${ }^{2}$ National Cancer Institute; 0.0. Bogomolets National Medical University; ${ }^{3}$ O.O. Bogomolets National Medical University

\subsection{6/ijgc-2020-ESG0.185}

Introduction/Background Vulvar malignant tumors are the rarest oncological disease of women's reproductive system in Ukraine. In 2018 there were registered only 477 new cases of vulvar cancer among all Ukrainian women. It is approximately three cases per 100 thousand among female population. However there are less common histological types, such as leiomyosarcoma, melanoma and lymphangiosarcoma, which make up approximately $1 \%$ of all cases. The main goal in surgical treatment is to obtain gross surgical margins, which sometimes require the usage of reconstructive techniques.

Methodology 